\title{
DECOMPOSITION FACTORS OF D-MODULES ON HYPERPLANE CONFIGURATIONS IN GENERAL POSITION
}

\author{
TILAHUN ABEBAW AND RIKARD BØGVAD \\ (Communicated by Lev Borisov) \\ Dedicated to the memory of Demissu Gemeda
}

\begin{abstract}
Let $\alpha_{1}, \ldots, \alpha_{m}$ be linear functions on $\mathbb{C}^{n}$ and $X=\mathbb{C}^{n} \backslash V(\alpha)$, where $\alpha=\prod_{i=1}^{m} \alpha_{i}$ and $V(\alpha)=\left\{p \in \mathbb{C}^{n}: \alpha(p)=0\right\}$. The coordinate ring $\mathcal{O}_{X}=\mathbb{C}[x]_{\alpha}$ of $X$ is a holonomic $A_{n}$-module, where $A_{n}$ is the $n$-th Weyl algebra, and since holonomic $A_{n}$-modules have finite length, $\mathcal{O}_{X}$ has finite length. We consider a "twisted" variant of this $A_{n}$-module which is also holonomic. Define $M_{\alpha}^{\beta}$ to be the free rank $1 \mathbb{C}[x]_{\alpha}$-module on the generator $\alpha^{\beta}$ (thought of as a multivalued function), where $\alpha^{\beta}=\alpha_{1}^{\beta_{1}} \ldots \alpha_{m}^{\beta_{m}}$ and the multi-index $\beta=\left(\beta_{1}, \ldots, \beta_{m}\right) \in \mathbb{C}^{m}$. It is straightforward to describe the decomposition factors of $M_{\alpha}^{\beta}$, when the linear functions $\alpha_{1}, \ldots, \alpha_{m}$ define a normal crossing hyperplane configuration, and we use this to give a sufficient criterion on $\beta$ for the irreducibility of $M_{\alpha}^{\beta}$, in terms of numerical data for a resolution of the singularities of $V(\alpha)$.
\end{abstract}

\section{INTRODUCTION}

Definition 1.1. Let $V \cong \mathbb{C}^{n}$ be a finite-dimensional complex vector space.

(i) A (finite) hyperplane configuration $A=\left\{H_{1}, \ldots, H_{m}\right\}$ in $V$ is a finite set of affine hyperplanes $H_{i}=V\left(\alpha_{i}\right)$ in $V$, where $\alpha_{i}: V \rightarrow \mathbb{C}$ is a non-zero polynomial of degree one.

(ii) A hyperplane configuration $A$ in $V$ is said to be in general position if

$$
\left\{H_{i_{1}}, \ldots, H_{i_{p}}\right\} \subset A, p \leq n \Longrightarrow \operatorname{dim}_{\mathbb{C}}\left(H_{i_{1}} \cap \ldots \cap H_{i_{p}}\right)=n-p
$$

and

$$
\left\{H_{i_{1}}, \ldots, H_{i_{p}}\right\} \subset A, p>n \Longrightarrow H_{i_{1}} \cap \ldots \cap H_{i_{p}}=\emptyset .
$$

(iii) A hyperplane configuration $A$ is said to be a normal crossing configuration if for any set of hyperplanes $H_{i_{1}}, \ldots, H_{i_{k}}$ that contain $p$, there is an affine change of local coordinates at $p$ to new coordinates $x_{1}, \ldots, x_{n}$, such that $\alpha_{i_{1}}=x_{1}, \ldots, \alpha_{i_{k}}=x_{k}$. In particular, any point $p \in V$ is contained in at most $n=\operatorname{dim} V$ of the hyperplanes in $A$.

If $A$ is a normal crossing configuration, then the divisor $\alpha=\prod_{i=1}^{m} \alpha_{i}$ is a normal crossings divisor (see [9]). Any configuration in general position is a normal crossing

Received by the editors July 14, 2010 and, in revised form, March 2, 2011.

2010 Mathematics Subject Classification. Primary 32C38, 52C35; Secondary 14F10, 32S22.

Key words and phrases. Hyperplane arrangements, D-module theory.

The first author was supported in part by the International Science Program, Uppsala University. 
configuration. The intersections $H \neq \mathbb{C}^{n}$ of a subset of the hyperplanes in $A$ are called the flats of the configuration.

Example 1.2. Let $V=\mathbb{C}^{2}$. Then a set of lines in $\mathbb{C}^{2}$ defines

(i) a hyperplane configuration in general position if no two are parallel and no three meet at a point and

(ii) a normal crossing configuration if no three meet at a point.

There is an extensive literature on hyperplane arrangements [16. The problem studied in this paper is the following. Let $A=\left\{H_{i}=V\left(\alpha_{i}\right), i=1, \ldots, m\right\}$, be a hyperplane configuration, and set $X=\mathbb{C}^{n} \backslash V(\alpha)$, where $\alpha=\prod_{i=1}^{m} \alpha_{i}$. The coordinate ring $\mathcal{O}_{X}$ of $X$ is the localization $\mathbb{C}[x]_{\alpha}:=\mathbb{C}\left[x_{1}, \ldots, x_{n}\right]_{\alpha}$ and this is a holonomic $A_{n}$-module, where $A_{n}$ is the $n$-th Weyl algebra. Consider the following twisted variant of this $A_{n}$-module, which corresponds to the multivalued function $\alpha^{\beta}=\alpha_{1}^{\beta_{1}} \ldots \alpha_{m}^{\beta_{m}}$.

Definition 1.3. The module $M_{\alpha}^{\beta}$ is (as a $\mathbb{C}[x]_{\alpha}$-module) the free rank $1 \mathbb{C}[x]_{\alpha^{-}}$ module on the generator $\alpha^{\beta}$, where $\beta=\left(\beta_{1}, \ldots, \beta_{m}\right) \in \mathbb{C}^{m}$. It is furthermore an $A_{n}$-module, defining

$$
\partial_{j}\left(\alpha^{\beta}\right)=\sum_{i=1}^{m} \beta_{i} \frac{\partial_{j}\left(\alpha_{i}\right)}{\alpha_{i}} \alpha^{\beta}
$$

for $j=1,2, \ldots, n$ and extending to an action of $A_{n}$ on $M_{\alpha}^{\beta}$.

The $A_{n}$-module $M_{\alpha}^{\beta}$ is holonomic and so has finite length. The decomposition factors have support on the flats associated to the configuration (Proposition 2.6). In continuation of [1], where we treated the case $n=2$, and inspired by [6], where incidentally the case of $\beta \in \mathbb{Z}^{m}$ is treated, our main interest lies in finding the decomposition factors of $M_{\alpha}^{\beta}$. If $A$ is a normal crossing configuration, this is not difficult. In Theorem 3.2 , we prove that in that case, $M_{\alpha}^{\beta}$ has exactly one decomposition factor with support on a flat $H=\bigcap_{k=1}^{r} H_{i_{k}}$ iff the associated $\beta_{i_{k}}, k=1, \ldots, r$ are integers. Normal crossing configurations occur when the singularities of $V(\alpha)$ are resolved. Applied to such a resolution, Theorem 3.2 together with the decomposition theorem for D-modules, then gives our main result, Theorem 5.4 a sufficient criterion for $M_{\alpha}^{\beta}$ to be irreducible for an arbitrary hyperplane arrangement, in terms of numerical data of the resolution and $\beta$.

D-modules on hyperplane configurations have been of interest to several authors, e.g., 14, 15, 18, not to mention many works on the corresponding equivalent category of sheaves. Khoroshkin and Varchenko (see [15]) study a subcategory of holonomic D-modules with regular singularities along the stratification given by the intersections of the hyperplanes and describe it in terms of quivers. This category however does not include our modules. We do not explicitly use Bernstein-Sato polynomials, though they clearly contain part of the information we are interested in, but it may be mentioned that they have been calculated for hyperplane configurations in, e.g., [18. The explicit presentation of the D-module submodule of $M_{\alpha}^{0}$ generated by $\alpha^{-1}$ has been studied in [2, 17] as part of a study of logarithmic differential forms.

As general references for D-module theory, we have used 4], [5], and [14. 


\section{Support of D-Modules}

2.1. Definition. Let $X$ be a smooth algebraic variety. We denote by $\mathcal{D}_{X}$ the sheaf of differential operators on $X$. If $X=\mathbb{C}^{n}$, this is the same as the sheaf on $\mathbb{C}^{n}$ associated to the Weyl algebra $A_{n}$. If $X$ is an affine variety, we will, by abuse of notation, identify $\mathcal{O}_{X}$ with $\Gamma\left(X, \mathcal{O}_{X}\right)$ and an $\mathcal{O}_{X}$-module sheaf with its global sections as a module over $\Gamma\left(X, \mathcal{O}_{X}\right)$. If $X$ is an affine open subset of $\mathbb{C}^{n}$ defined by $0 \neq \alpha \in \mathbb{C}[x]$, then $\mathcal{O}_{X}=\mathbb{C}[x]_{\alpha}$ and $\mathcal{D}_{X}=\mathbb{C}[x]_{\alpha} \otimes_{\mathbb{C}[x]} A_{n}$.

A holonomic $\mathcal{D}_{X}$-module $M$ is in particular an $\mathcal{O}_{X}$-module and as such may be shown to have support on a closed variety $Z=\operatorname{Supp} M$ (see [3]). If $\mathcal{I} \subset \mathcal{O}_{X}$ is the ideal associated to $Z$, any local section of $M$ is annihilated by a large enough power of $\mathcal{I}$, and $Z$ is the minimal closed subset with this property.

We will see later that the support of the irreducible factors of $M_{\alpha}^{\beta}$ consists of intersections of hyperplanes.

Example 2.1. $\quad$ (i) Let $M_{1}=\mathbb{C}[x, y]_{x y} /\left(\mathbb{C}[x, y]_{x}+\mathbb{C}[x, y]_{y}\right)$. Then

$$
\operatorname{Supp} M_{1}=V(x, y)=(0,0) \text {. }
$$

(ii) Let $M_{2}=\mathbb{C}[x, y]_{x} / \mathbb{C}[x, y]$. Then $\operatorname{Supp} M_{2}=V(x)=\{(0, y): y \in \mathbb{C}\}$.

(iii) Let $M_{3}=\mathbb{C}[x, y]$. Then $\operatorname{Supp} M_{3}=V(0)=\mathbb{C}^{2}$.

2.2. Some basic properties. Let $M$ be a $\mathcal{D}_{X}$-module and $U \subset X$ be an open subset. Then define $M_{\left.\right|_{U}}=: \mathcal{O}_{U} \otimes_{\mathcal{O}_{X}} M$; this is a $\mathcal{D}_{U}$-module.

Lemma 2.2. Let $U \subset X$ be an open subset.

(i) $\operatorname{Supp} M_{\left.\right|_{U}}=U \cap \operatorname{Supp} M$.

(ii) $M_{\left.\right|_{U}}=0 \Leftrightarrow \operatorname{Supp} M \subset X-U=: Z$.

Proof. (i) is clear by the above description. Let $\mathcal{I}$ be the ideal of a closed subvariety $Z$, and let $j: U \rightarrow X$ be the inclusion. For any $\mathcal{O}_{X}$-module $\mathcal{M}$ there exists an exact sequence of $\mathcal{O}_{X}$-modules

$$
\Gamma_{Z} M \subset M \longrightarrow j_{*} M_{\left.\right|_{U}}
$$

where (locally) $\Gamma_{Z} M=\left\{m \in M: \exists r, \mathcal{I}^{r} m=0\right\}$. If $M_{\left.\right|_{U}}=0$, then $\Gamma_{Z} M=M$ and this proves (ii) in one direction. The other direction is immediate.

It is easy to see that localization preserves irreducibility of $\mathcal{D}_{X}$-modules. (Cf. also Lemmas 2.1-2 of [11]; since the formulation there is different and without proof, we give one.)

Proposition 2.3. If $M$ is an irreducible $\mathcal{D}_{X}$-module and $U \subset X$ an open subset, then $M_{\left.\right|_{U}}=: \mathcal{O}_{U} \otimes_{\mathcal{O}_{X}} M$ is an irreducible $\mathcal{D}_{U}$-module.

Proof. Let $j: U \rightarrow X$ be the inclusion and denote the adjunction in (2.1) by $\delta: M \rightarrow$ $j_{*} M_{\left.\right|_{U}}$. Since $M$ is irreducible, $\delta$ is either 0 or an injection. In the first case $M$ is $Z$-torsion and $\left.M\right|_{U}=0$, and the lemma is trivially true. So assume that $\delta$ is an injection, and that $K \subset M_{\left.\right|_{U}}$ is an irreducible $\mathcal{D}_{U}$-submodule. Then we have an injection $\gamma: j_{*} K \rightarrow j_{*} M_{\left.\right|_{U}}$. The intersection $\delta(M) \cap \gamma\left(j_{*} K\right)$ is either 0 or $\delta(M)$. In the second case $\delta(M) \subset \gamma\left(j_{*} K\right)$. By restricting to $U$ and using that $\left.\delta\right|_{U}$ is the identity and $\left.\gamma\right|_{U}$ is the inclusion $K \subset M_{\left.\right|_{U}}$, we get that $\left.M\right|_{U}=K$. Hence the lemma is true in this case too. The first case implies that $\gamma: j_{*} K \rightarrow j_{*} M_{\left.\right|_{U}} / \delta(M)$ is an inclusion, which implies that $j_{*} K$ is a $Z$-torsion module. Hence $K=\left.\left(j_{*} K\right)\right|_{U}=$ 0 . 
Since localization is an exact functor we have the following corollary. We will use the notation $D F(M)$ for the set of decomposition factors of $M$ and $c(M)$ for the number of decomposition factors.

Corollary 2.4. $\quad$ (i) $D F\left(M_{\left.\right|_{U}}\right)=\left\{M_{i} \in D F(M): \operatorname{Supp} M_{i} \cap U \neq \emptyset\right\}$.

(ii) $c\left(M_{\left.\right|_{U}}\right) \leq c(M)$.

2.3. The support of decomposition factors of $M_{\alpha}^{\beta}$. That the support of decomposition factors of the modules we study consists of intersections of hyperplanes is easily seen by general arguments, but it is also possible to give a direct argument, as we will now do. First we will do a reduction.

Let $V_{1}=\bigcap_{i=1}^{m} H_{i}$ and assume that this affine space has positive dimension. Choose a vector space complement $V_{2}$ to the affine subspace $V_{1}$, such that $\mathbb{C}^{n} \cong$ $V_{1} \oplus V_{2}$, and denote the restriction of a linear function $\alpha$ to $V_{2}$ by $\tilde{\alpha}$.

Lemma 2.5 (Reduction lemma).

i) $M_{\alpha}^{\beta}$ is isomorphic to the exterior tensor product $M_{\tilde{\alpha}}^{\beta} \widehat{\otimes} \mathcal{O}_{V_{1}}$, as $A_{n}$-modules.

ii) If $N_{i}, i=1, \ldots, r$ are the decomposition factors of $M_{\tilde{\alpha}}^{\beta}$, then $N_{i} \widehat{\otimes} \mathcal{O}_{V_{1}}, i=$ $1, \ldots, r$ are the decomposition factors of $M_{\alpha}^{\beta}$.

iii) $\operatorname{Supp}\left(N_{i} \widehat{\otimes} \mathcal{O}_{V_{1}}\right)=\left(\operatorname{Supp} N_{i}\right) \times V_{1}, i=1, \ldots, r$.

Proof. i) follows by an affine change of coordinates. By Corollary 2.5 of [1] a decomposition factor of $M_{\alpha}^{\beta}$ is the external tensor product of a decomposition factor of $M_{\tilde{\alpha}}^{\beta}$ with $\mathcal{O}_{V_{1}}$. This gives ii). Finally, iii) is immediate.

Proposition 2.6. Decomposition factors of $M_{\alpha}^{\beta}$ have support on intersections of hyperplanes.

Proof. Use the notation $H_{S}=\left\{p \in \mathbb{C}^{n}: \alpha_{i}(p)=0, i \in S\right\}$, where $S \subset\{1,2, \ldots, m\}$. By the lemma we can reduce to the case when $V_{1}$ is a point or $V_{1}=\emptyset$. Make an induction on the number $m$ of hyperplanes; for $m=0$ the statement is immediate from the irreducibility of $\mathbb{C}[x]$ as an $A_{n}$-module. Assume it is true for an arbitrary arrangement with $m$ hyperplanes. Then we have to prove the following: if $N$ is a decomposition factor of $M_{\alpha}^{\beta}$, where $\alpha=\prod_{i=1}^{m+1} \alpha_{i}$, then $H:=\operatorname{Supp} N$ is an intersection of hyperplanes.

By our reduction, there exists a hyperplane $H_{j}$ such that $H \nsubseteq H_{j}$, except in the case where $H$ equals $\bigcap_{i=1}^{m+1} H_{i}$, and so in particular is a hyperplane intersection, and there is nothing to prove. Let $U=\mathbb{C}^{n} \backslash H_{j}$. Then by Proposition 2.3. $\left.N\right|_{U}$ is non-zero and so a decomposition factor of $\left.M_{\alpha}^{\beta}\right|_{U}$. On $U, \alpha_{j}$ is invertible and by Lemma 2.9 below, $\gamma:\left.\left.M_{\alpha}^{\beta}\right|_{U} \cong M_{\bar{\alpha}}^{\bar{\beta}}\right|_{U}$, where $\bar{\alpha}=\prod_{i=1, i \neq j}^{m+1} \alpha_{i}$ and $\bar{\beta}=\left(\beta_{1}, \ldots \widehat{\beta}_{j}, \ldots, \beta_{m+1}\right)$. There is, again by Proposition 2.3 a decomposition factor $N_{1}$ of $M_{\bar{\alpha}}^{\bar{\beta}}$, such that $\left.N_{1}\right|_{U}=\gamma\left(\left.N\right|_{U}\right)$. By induction, $\operatorname{Supp} N_{1}=H_{S}$ is an intersection of hyperplanes. Since twisting modules by automorphisms as in Lemma 2.9 preserves support, $\operatorname{Supp} N \cap U=H_{S} \cap U$. Since $N$ is irreducible, $\operatorname{Supp} N$ is an irreducible subvariety, as is $H_{S}$, and so $\operatorname{Supp} N=\overline{\operatorname{Supp}} N \cap U=\overline{H_{S} \cap U}=H_{S}$. This proves the induction step.

Definition 2.7. Suppose that $\theta$ is an automorphism of $\mathcal{D}_{X}$. If $M$ is a $\mathcal{D}_{X}$-module, $\theta^{*} M$ is defined to be the $\mathcal{D}_{X}$-module which consists locally of the same sections as $M$, but on which (local sections of) $\mathcal{D}_{X}$ acts by $\theta$ : if $P \in \mathcal{D}_{X}, m \in \theta^{*} M$, then $P m=\theta(P) m$. 
The following lemma is clear.

Lemma 2.8. Let $\theta: \mathcal{D}_{X} \longrightarrow \mathcal{D}_{X}$ denote an automorphism that is the identity on $\mathcal{O}_{X}$. Suppose $M$ has decomposition factors $M_{i}, i=1, \ldots, l$. Then $\theta^{*} M$ has decomposition factors $\theta^{*} M_{i}, i=1, \ldots, l$. In particular $c(M)=c\left(\theta^{*} M\right)$. The support of $\theta^{*} M_{i}$ equals the support of $M_{i}$.

We apply this in the following situation.

Proposition 2.9. Suppose that $X=\mathbb{C}^{n}$, so that $\mathcal{O}_{X}=\mathbb{C}[x]$ and let $U=X$ $V\left(\alpha_{1}, \ldots, \alpha_{l}\right)$. Then $c\left(M_{\alpha \mid U}^{\beta}\right)=c\left(M_{\bar{\alpha} \mid U}^{\bar{\beta}}\right)$, where $\tilde{\alpha}=\alpha_{l+1} \ldots \alpha_{m}$ and $\tilde{\beta}=\left(\beta_{l+1}, \ldots\right.$, $\left.\beta_{m}\right)$.

Proof. By the preceding lemma, it suffices to construct an automorphism $\theta: \mathcal{D}_{\mathcal{U}} \rightarrow$ $\mathcal{D}_{\mathcal{U}}$ that is the identity on $\mathcal{O}_{U}$ and such that $\left.\theta^{*}\left(\left.M_{\beta}^{\beta}\right|_{U}\right) \cong M_{\bar{\alpha}}^{\bar{\beta}}\right|_{U}$. Further it is by induction enough to assume that $U=X-V\left(\alpha_{1}\right)$. Put $\alpha^{\beta}=\alpha_{1}^{\beta_{1}} \tilde{\alpha}^{\tilde{\beta}}$, where $\tilde{\alpha}=\alpha_{2} \ldots \alpha_{m}$ and $\tilde{\beta}=\left(\beta_{2}, \ldots, \beta_{m}\right)$. Then $\left.M_{\alpha}^{\beta}\right|_{U}=\mathbb{C}[x]_{\alpha} \alpha_{1}^{\beta_{1}} \tilde{\alpha}^{\tilde{\beta}}$ where $\alpha_{1}$ is invertible. Define $\theta: \mathcal{D}_{\mathcal{U}} \longrightarrow \mathcal{D}_{\mathcal{U}}$ by

$$
\theta(D)=D+\frac{\beta_{1} D\left(\alpha_{1}\right)}{\alpha_{1}}
$$

for all $D \in \operatorname{Der}_{\mathbb{C}}(\mathbb{C}[x]) \subset \mathcal{D}_{\mathcal{U}}$ and $\theta(r)=r$ for all $r \in \mathcal{O}_{U}$, and extend this to an endomorphism of $\mathcal{D}_{\mathcal{U}}$. This is the desired automorphism, since it is easily checked that it has an inverse and that the map

$$
\rho:\left.\theta^{*}\left(\left.M_{\tilde{\alpha}}^{\tilde{\beta}}\right|_{U}\right) \longrightarrow M_{\alpha}^{\beta}\right|_{U},
$$

defined by $\rho\left(r \tilde{\alpha}^{\tilde{\beta}}\right)=r \alpha^{\beta}$, is a $\mathcal{D}_{\mathcal{U}}$-isomorphism.

\section{DECOMPOSITION FACTORS ON NORMAL CROSSINGS}

We will now describe the decomposition factors of $M_{\alpha}^{\beta}$ for a normal crossing hyperplane configuration.

Lemma 3.1. Let $A=\left\{H_{1}, \ldots, H_{m}\right\}$ be a non-empty normal crossing hyperplane configuration in $\mathbb{C}^{n}$. If $\bigcap_{i=1}^{m} H_{i} \neq \emptyset$, then $m \leq n$ and the arrangement is central. Assume that $\bigcap_{i=1}^{m} H_{i}=\emptyset$. Then, for each intersection $H\left(\neq \mathbb{C}^{n}\right)$ of a subset of the hyperplanes in $A$,

(i) there exists at least one hyperplane $H_{i}$, such that $H \nsubseteq H_{i}$, and

(ii) there is a unique subset $S=\left\{i_{1}, \ldots, i_{k}\right\} \subset\{1, \ldots, m\}$ such that $H=H_{S}=$ $\bigcap_{k=1}^{r} H_{i_{k}}$. Furthermore $H_{j} \supset H \Longleftrightarrow j \in S$.

The following theorem, together with the fact that all decomposition factors have support on intersections of hyperplanes (Lemma 2.6), gives the number of decomposition factors of $M_{\alpha}^{\beta}$ and their support.

Theorem 3.2. Let $A=\left\{H_{1}, \ldots, H_{m}\right\}$ be a normal crossing arrangement on $\mathbb{C}^{n}$. Let $H_{S}=H_{i_{i}} \cap \ldots \cap H_{i_{k}}$ be a flat. Consider decomposition factors of $M_{\alpha}^{\beta}$.

(i) There is at most one decomposition factor with support on $H_{S}$.

(ii) There is exactly one decomposition factor with support on $H_{S}$ if and only if $\beta_{i_{1}}, \ldots, \beta_{i_{k}} \in \mathbb{Z}$. 
Proof. By Lemma 2.5 we may assume that $\bigcap_{i=1}^{m} H_{i}$ is either a point or $\emptyset$. If this intersection is a point $p$, then as stated in Lemma 3.1, the arrangement is central and $m \leq n$, and the theorem is easy to prove; see [1, Prop. 3.1]. (The idea is that by a basis change it may be assumed that $\alpha_{i}=x_{i}, i=1, \ldots, m$ are coordinates at $p$, and then $M_{\alpha}^{\beta}$ is isomorphic to the external tensor product of $M_{\alpha_{i}}^{\beta_{i}} i=1, \ldots, m$ on $\mathbb{C}^{1}$.)

This gives the induction basis for an inductive proof of (i) and (ii) on the number of hyperplanes in the arrangement, and it also allows us to assume that the intersection of all the hyperplanes in our configuration is empty. Assume that (i) and (ii) are true for all normal crossing arrangements with $m$ hyperplanes, and let $A=\left\{H_{i}\right\}_{i=1}^{m+1}$ be a normal crossing arrangement with $m+1$ hyperplanes.

(i) For $H_{S}=\mathbb{C}^{n}$ the statement of (i) follows trivially, since $M_{\alpha}^{\beta}$ has rank 1 as a module over $O_{U}$, where $U=\mathbb{C}^{n} \backslash V(\alpha)$. Let $H_{S} \neq \mathbb{C}^{n}$ be a flat of $A$. By Lemma 3.1 there is a hyperplane, which we may assume is $H_{m+1}$, such that $H_{S} \nsubseteq H_{m+1}$. Let $U=X \backslash H_{m+1}$. By Proposition 2.3 any decomposition factor $F$ of $M_{\alpha}^{\beta}$ with $\operatorname{Supp} F=H$ satisfies that the restriction $\left.F\right|_{U}$ is a non-zero irreducible $D_{U}$-module. By the proof of Lemma 2.9, the $D_{U}$-module $\left.M_{\alpha}^{\beta}\right|_{U}$ is isomorphic to the module $\left.M_{\tilde{\alpha}}^{\tilde{\beta}}\right|_{U}$ twisted by an automorphism, where $\tilde{\alpha}=\alpha_{1} \ldots \alpha_{m}$ and $\tilde{\beta}=\left(\beta_{1}, \ldots, \beta_{m}\right)$. The twisting does not change the number of decomposition factors nor their support. By the induction assumption and Proposition $2.3,\left.M_{\tilde{\alpha}}^{\tilde{\beta}}\right|_{U}$ has at most one decomposition factor on $H_{S} \cap U$. So the same is true of $M_{\alpha}^{\beta}$ and by induction (i) is proved.

(ii) Similarly, we can use induction to prove one direction of (ii). Assume that (ii) is true for all normal crossing configurations with $m$ hyperplanes, and that we have an arrangement $\left\{H_{i}\right\}_{i=1}^{m+1}$. Let $H=H_{j_{1}} \cap \ldots \cap H_{j_{s}}$ be a flat such that $\beta_{j_{1}} \in \mathbb{C} \backslash \mathbb{Z}$. By Lemma 3.1 there exists $i$ such that $H \nsubseteq H_{i}$. By $(i)$ there exists at most one decomposition factor of $M_{\alpha}^{\beta}$ with support $H$. Assume that there exists a decomposition factor of $M_{\alpha}^{\beta}$, say $F$, with $\operatorname{Supp} F=H$. Let $U=\mathbb{C}^{n} \backslash H_{i}$. Then $\left.F\right|_{U}$ is simple and $\left.\operatorname{Supp} F\right|_{U}=H \cap U$. Let $\tilde{\alpha}=\alpha_{1} \ldots \hat{\alpha}_{i} \ldots \alpha_{m+1}$ and $\tilde{\beta}=\left(\beta_{1}, \ldots, \hat{\beta}_{i}, \ldots, \beta_{m+1}\right)$. Again arguing as in the proof of (i), we get that $M_{\tilde{\alpha}}^{\tilde{\beta}}$ has a decomposition factor with support on $H$. But this is a contradiction to the induction assumption. Therefore there is no decomposition factor of $M_{\alpha}^{\beta}$ which has support on $H$.

The converse of (ii) may be proven directly. Without loss of generality assume that $H=\bigcap_{i=1}^{k} H_{i}$ such that $\beta_{1}, \ldots, \beta_{k} \in \mathbb{Z}$. Since we have a normal crossings arrangement, we may, by affine base change, assume that $\alpha_{i}=$ $x_{i}, i=1, \ldots, k$. Then $M_{\alpha}^{\beta} \cong \mathbb{C}[x]_{\alpha} \tilde{\alpha}^{\tilde{\beta}}$, where $\tilde{\alpha}^{\tilde{\beta}}=\alpha_{k+1}^{\beta_{k+1}} \ldots \alpha_{m}^{\beta_{m}}$. We first prove that there is one subfactor of $M_{\alpha}^{\beta}$ with support on $H$. Let $N \subset \mathbb{C}[x]_{\alpha}$ be the vector space over $\mathbb{C}$ generated by the set

$$
\left\{\prod_{j=1}^{n} x_{j}^{r_{j}} \prod_{l=k+1}^{m} \alpha_{l}^{s_{l}} \tilde{\alpha}^{\tilde{\beta}}: r_{j} \geq 0 \text { if } j=k+1, \ldots, n\right\} .
$$

As a $\mathbb{C}[x]$-module this is isomorphic to the localization of $\mathbb{C}[x]_{x_{1} \ldots x_{k}}$ to the open subset $\mathbb{C}^{n} \backslash \bigcup_{j=k+1}^{m} H_{j}$. It is clearly an $A_{n}$-module. Further let 
$N^{+} \subset N$ be the vector space over $\mathbb{C}$ generated by the set

$\left\{\prod_{i=1}^{n} x_{j}^{r_{j}} \prod_{l=k+1}^{m} \alpha_{l}^{s_{l}} \tilde{\alpha}^{\tilde{\beta}}: r_{k+1} \geq 0, \ldots, r_{n} \geq 0 \& \exists l: 1 \leq l \leq k \& r_{l} \geq 0\right\}$.

As a $\mathbb{C}[x]$-module this is isomorphic to the localization of $\sum_{j=1}^{k} \mathbb{C}[x]_{x_{1} \ldots \hat{x}_{j} \ldots x_{k}}$ to $\mathbb{C}^{n} \backslash \bigcup_{j=k+1}^{m} H_{j}$. Again it is clearly an $A_{n}$-module, and $N / N^{+}$is nonzero, since the quotient

$$
\mathbb{C}[x]_{x_{1} \ldots x_{k}} /\left(\sum_{j=1}^{k} \mathbb{C}[x]_{x_{1} \ldots \hat{x}_{j} \ldots x_{k}}\right)
$$

is nonzero, with support on $H$, and $H \cap \mathbb{C}^{n} \backslash \bigcup_{j=k+1}^{m} H_{j} \neq \emptyset$, by Lemma 3.1 (ii). Then $N / N^{+}$is a non-zero $A_{n}$-module, which is a decomposition factor of $M_{\alpha}^{\beta}$. As noted above, $\operatorname{Supp}\left(N / N^{+}\right)=V\left(\alpha_{1}, \ldots, \alpha_{k}\right)=\bigcap_{i=1}^{k} H_{i}=H$, and hence there must be at least one irreducible decomposition factor with support on $H$.

This completes the proof of the theorem.

It should be noted that the proof uses only that $\alpha_{i}$ such that the $\alpha_{i}(p)=0$ form part of a system of parameters at each point $p$ and hence works in this situation for an arbitrary smooth affine variety.

Definition 3.3. Let A be a hyperplane configuration in $\mathbb{C}^{n}$ and $\mathcal{H}$ be the set of all non-empty intersections of hyperplanes in A, including $\mathbb{C}^{n}$ itself considered as the intersection over the empty set. Define a relation $x \leq y$ in $\mathcal{H}$ if $x \supseteq y$ (as subsets of $\mathbb{C}^{n}$ ). In other words, $\mathcal{H}$ is partially ordered by reverse inclusion. We call $\mathcal{H}$ the intersection poset of $\mathrm{A}$.

We cannot give formulas for the cardinality $|\mathcal{H}|$ valid for all normal crossings arrangements, but in the special case when $\mathrm{A}$ is a hyperplane configuration in general position, this is possible. In that case any intersection of less than or equal to $n$ hyperplanes gives a flat (see [13]):

$$
|\mathcal{H}|=\sum_{k=0}^{n}\left(\begin{array}{l}
m \\
k
\end{array}\right) \text {. }
$$

Corollary 3.4. Let $M_{\alpha}^{\beta}=\mathbb{C}[x]_{\alpha} \alpha^{\beta}$, where $\alpha^{\beta}=\alpha_{1}^{\beta_{1}} \ldots \alpha_{m}^{\beta_{m}}$ and the set $\left\{\alpha_{1}, \ldots, \alpha_{m}\right\}$ defines a hyperplane configuration in general position. Assume that $\beta_{1}, \ldots, \beta_{k} \in \mathbb{Z}$ and $\beta_{k+1}, \ldots, \beta_{m} \in \mathbb{C} \backslash \mathbb{Z}$. Then the number of decomposition factors of $M_{\alpha}^{\beta}, c\left(M_{\alpha}^{\beta}\right)$, is given by

$$
c\left(M_{\alpha}^{\beta}\right)=\sum_{j=0}^{n}\left(\begin{array}{l}
k \\
j
\end{array}\right)
$$

where $\left(\begin{array}{l}k \\ j\end{array}\right)=0$ if $j>k$.

Proof. In Theorem 3.2 we proved that $M_{\alpha}^{\beta}$ has exactly one decomposition factor for each flat $H$, where $H=H_{i_{1}} \cap \ldots \cap H_{i_{s}}$ and $\beta_{i_{1}}, \ldots, \beta_{i_{s}} \in \mathbb{Z}$. The sum in the theorem clearly counts the number of those, keeping in mind that by the hypothesis on the arrangement, each non-empty subset $S \subset\{1, \ldots, m\}$ corresponds to a unique flat.

An example is given in the next section. 

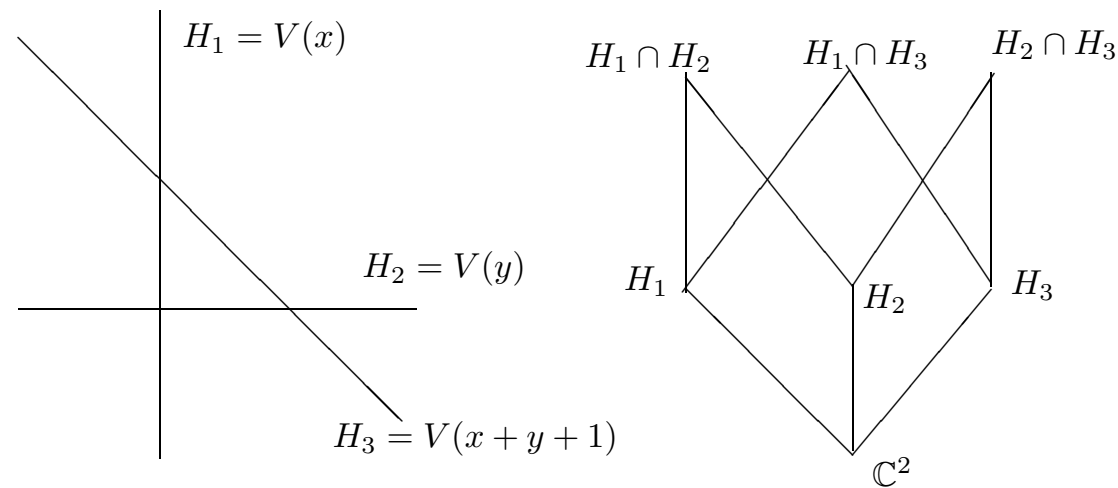

FiguRE 1. A hyperplane configuration defined by the polynomial $\alpha=x y(x+y+1)$ and the Hasse diagram of its intersection poset.

\section{EXAMPLE}

4.1. The $A_{2}$-module $M_{\alpha}^{\beta}=\mathbb{C}[x, y]_{x y(x+y+1)} x^{\beta_{1}} y^{\beta_{2}}(x+y+1)^{\beta_{3}}$. According to the previous theorem, the number of decomposition factors is $1,2,4$ or $7=\left(\begin{array}{l}3 \\ 0\end{array}\right)+$ $\left(\begin{array}{l}3 \\ 1\end{array}\right)+\left(\begin{array}{l}3 \\ 2\end{array}\right)$, according to the number of $\beta_{i} \in \mathbb{Z}$ being $0,1,2$ or 3 . We will describe the decomposition factors of $M_{\alpha}^{\beta}$ explicitly for these different cases, as an example for the previous section. Even though this is a plane arrangement, it is not covered by [1, since the arrangement is not central. See Figure 1 for the Hasse diagram of the corresponding flats. The following corollary summarizes the results.

Corollary 4.1. Consider the $A_{2}$-module

$$
M_{\alpha}^{\beta}=\mathbb{C}[x, y]_{x y(x+y+1)} x^{\beta_{1}} y^{\beta_{2}}(x+y+1)^{\beta_{3}} .
$$

(i) If $\beta_{1}, \beta_{2}, \beta_{3} \in \mathbb{C} \backslash \mathbb{Z}$, then $c\left(M_{\alpha}^{\beta}\right)=1$ and hence $M_{\alpha}^{\beta}$ is irreducible.

(ii) If $\beta_{1} \in \mathbb{Z}$ and $\beta_{2}, \beta_{3} \in \mathbb{C} \backslash \mathbb{Z}$, then $c\left(M_{\alpha}^{\beta}\right)=2$.

(iii) If $\beta_{1}, \beta_{2} \in \mathbb{Z}$ and $\beta_{3} \in \mathbb{C} \backslash \mathbb{Z}$, then $c\left(M_{\alpha}^{\beta}\right)=4$.

(iv) If $\beta_{1}, \beta_{2}, \beta_{3} \in \mathbb{Z}$, then $c\left(M_{\alpha}^{\beta}\right)=7$.

Proof. The linear functions $x, y$ and $x+y+1$ define a general position hyperplane configuration in $\mathbb{C}^{2}$. Therefore, by the previous section, the number of the decomposition factors of $M_{\alpha}^{\beta}$ is the number of flats $H$ such that the corresponding $\beta$ 's of the linear functions of the hyperplanes defining $H$ are integers.

(i) If $\beta_{1}, \beta_{2}, \beta_{3} \in \mathbb{C} \backslash \mathbb{Z}$, then $H=\mathbb{C}^{2}$, considered as the empty intersection, is the only flat such that the $\beta$ 's of the linear functions defining the hyperplanes defining $\mathbb{C}^{2}$ are integers. Therefore $M_{\alpha}^{\beta}$ has only one decomposition factor with support on $\mathbb{C}^{2}$, and hence $M_{\alpha}^{\beta}$ is irreducible with support on $\mathbb{C}^{2}$.

(ii) If $\beta_{1} \in \mathbb{Z}$ and $\beta_{2}, \beta_{3} \in \mathbb{C} \backslash \mathbb{Z}$, then $H_{1}=\mathbb{C}^{2}$, considered as the empty intersection and $H_{2}=V(x)$ are the flats with corresponding integer $\beta$ 's. We can also see this by the following composition series:

$$
\mathbb{C}[x, y]_{y(x+y+1)} y^{\beta_{2}}(x+y+1)^{\beta_{3}} \subset \mathbb{C}[x, y]_{x y(x+y+1)} x^{\beta_{1}} y^{\beta_{2}}(x+y+1)^{\beta_{3}},
$$


where

$$
\mathbb{C}[x, y]_{y(x+y+1)} y^{\beta_{2}}(x+y+1)^{\beta_{3}}
$$

and

$\mathbb{C}[x, y]_{x y(x+y+1)} x^{\beta_{1}} y^{\beta_{2}}(x+y+1)^{\beta_{3}} / \mathbb{C}[x, y]_{y(x+y+1)} y^{\beta_{2}}(x+y+1)^{\beta_{3}}$

are easily seen to be irreducible $A_{2}$-modules. Therefore, as predicted, $c\left(M_{\alpha}^{\beta}\right)=2$, with one decomposition factor with support on $V(x)$ and one decomposition factor with support on the whole space $\mathbb{C}^{2}$.

(iii) If $\beta_{1}, \beta_{2} \in \mathbb{Z}$ and $\beta_{3} \in \mathbb{C} \backslash \mathbb{Z}$, then $H_{1}=\mathbb{C}^{2}$, considered as the empty intersection and $H_{2}=V(x), H_{3}=V(y)$ and $H_{4}=V(x, y)$ are the flats with corresponding integer $\beta$ 's. Consider the following sequence of vector spaces over $\mathbb{C}$ :

$$
R_{0} \subset R_{1} \subset M_{\alpha}^{\beta}
$$

where $R_{0}=\mathbb{C}[x, y]_{(x+y+1)}(x+y+1)^{\beta_{3}}$, and $R_{1}$ is generated by the set

$$
\left\{\frac{\alpha_{j}^{r_{j}}(x+y+1)^{s}}{\alpha_{i}^{s_{i}}(x+y+1)^{\beta_{3}}}: s_{j} \geq 0, s_{i} \geq 1, \text { for } i, j=1,2\right\} .
$$

Therefore $c\left(M_{\alpha}^{\beta}\right)=4$ with one decomposition factor with support on each $H_{2}, H_{3}, H_{4}$, one decomposition factor with support on $\mathbb{C}^{2}$.

(iv) If $\beta_{1}, \beta_{2}, \beta_{3} \in \mathbb{Z}$, then $H_{1}=\mathbb{C}^{2}$, considered as the empty intersection and $H_{2}=V(x), H_{3}=V(y), H_{4}=V(x+y+1), H_{5}=V(x, y), H_{6}=V(x, x+$ $y+1)$ and $H_{7}=V(y, x+y+1)$ are the flats with their $\beta$ 's of the linear functions defining the hyperplanes where $H_{1}, \ldots, H_{7}$ are integers. Therefore $c\left(M_{\alpha}^{\beta}\right)=7$, with exactly one decomposition factor with support on each of the flats.

\section{Resolution of Singularities}

Let $X=\mathbb{C}^{n} \backslash V(\alpha)$, and $\pi: \tilde{\mathbb{C}}^{n} \rightarrow \mathbb{C}^{n}$ be a resolution of the singularities of $V(\alpha)$. Let $E=\bigcup_{i=1}^{s} E_{i}$, where the $E_{i}$ are irreducible, be the exceptional divisor, and let $Z=\pi(E)$ be the center, so that $\tilde{\mathbb{C}}^{n} \backslash E \cong \mathbb{C}^{n} \backslash Z$. We then have that

$$
\operatorname{Div} \pi^{*}\left(\alpha_{i}\right)=\tilde{H}_{i}+r_{1}^{i} E_{1}+\ldots+r_{s}^{i} E_{s},
$$

where $\tilde{H}_{i}$ is the proper transform of $H_{i}=V\left(\alpha_{i}\right)$ and $r_{j}^{i} \in \mathbb{Z}_{+}$. Hence in an open affine $U \subset \tilde{\mathbb{C}}^{n}$,

$$
\left.\pi^{*}\left(\alpha_{i}\right)\right|_{U}=\left(\tilde{\alpha}_{i}^{U}\left(\gamma_{1}^{U}\right)^{r_{1}^{i}} \ldots\left(\gamma_{s}^{U}\right)^{r_{s}^{i}}\right) \subset \mathcal{O}_{U},
$$

where $\tilde{H}_{i} \cap U=V\left(\tilde{\alpha}_{i}^{U}\right)$ and $U \cap E_{j}=V\left(\gamma_{j}^{U}\right)$. Some of the intersections $\tilde{H}_{i} \cap U$ or $U \cap E_{j}$ may be empty, but we suppress this from the notation so as to not make it cumbersome. The properties of $\tilde{\mathbb{C}}^{n}$ that we need are:

i) that there is an affine cover $\left\{U_{i} \subset \tilde{\mathbb{C}}^{n}, i=1, \ldots, r\right\}$, where each $U=U_{i}$ is isomorphic to an open affine subvariety of finite type of $\mathbb{C}^{n}$;

ii) $\pi^{-1} V(\alpha)$ is a normal crossing divisor.

For these standard properties, see e.g. 9] or the construction in the present case in [7. We will use the preceding section to study the D-module pullback $\pi^{*} M_{\alpha}^{\beta}$. The last statement above implies that Theorem 3.2 applies to the restriction of this module to each $U_{i}$. 
Lemma 5.1. Let $U=U_{i}, i=1, \ldots, r$. Then $\left.\pi^{*} M_{\alpha}^{\beta}\right|_{U} \cong M_{\tilde{\alpha}}^{\tilde{\beta}}$ as $D_{U}$-modules, where $\tilde{\alpha}=\tilde{\alpha}_{1}^{U} \ldots . \tilde{\alpha}_{m}^{U} \gamma_{1}^{U} . . \gamma_{s}^{U}$. (In the product we use only the factors for which the corresponding intersections $\tilde{H}_{i} \cap U$ or $U \cap E_{j}$ are non-empty.) Further (with the same restriction)

$$
\left(\tilde{\beta}_{1}, \tilde{\beta}_{2}, \ldots, \tilde{\beta}_{m+s}\right)=\left(\beta_{1}, \beta_{2}, \ldots, \beta_{m}, \sum_{i=1}^{m} r_{1}^{i} \beta_{i}, \ldots, \sum_{i=1}^{m} r_{s}^{i} \beta_{i}\right) .
$$

Using the last section, we immediately obtain the decomposition factors of $\pi^{*} M_{\alpha}^{\beta}$. Extend the notation by defining $\tilde{H}_{m+i}:=E_{i}, i=1, \ldots, s$.

Corollary 5.2. The module $\pi^{*}\left(M_{\alpha}^{\beta}\right)$ has exactly one decomposition factor for each flat $\tilde{H}=\tilde{H}_{i_{1}} \cap \ldots \cap \tilde{H}_{i_{k}}$ such that $\tilde{\beta}_{i_{1}}, \ldots, \tilde{\beta}_{i_{k}} \in \mathbb{Z}$.

Proof. Since the $U_{i}$ cover $\tilde{\mathbb{C}}^{n}$, there is to any decomposition factor $N$ of $\pi^{*}\left(M_{\alpha}^{\beta}\right)$ a $U_{i}$ such that $\left.N\right|_{U_{i}} \neq 0$. This restriction has, by Proposition 2.6. support on an intersection $\tilde{H} \cap U_{i}=\tilde{H}_{i_{1}} \cap \ldots \cap \tilde{H}_{i_{k}} \cap U_{i}$, and by Theorem 3.2 and the preceding lemma, this implies that $\tilde{\beta}_{i_{1}}, \ldots, \tilde{\beta}_{i_{k}} \in \mathbb{Z}$. Furthermore there is by Theorem 3.2 at most one. Conversely, assume that $\tilde{H}=\tilde{H}_{i_{1}} \cap \ldots \cap \tilde{H}_{i_{k}}$ is such that $\tilde{\beta}_{i_{1}}, \ldots, \tilde{\beta}_{i_{k}} \in$ $\mathbb{Z}$. There is a $U_{i}$ such that $\tilde{H} \cap U_{i} \neq \emptyset$, and hence there is by Theorem 3.2 a decomposition factor of $\left.\pi^{*}\left(M_{\alpha}^{\beta}\right)\right|_{U_{i}}$ with support on $\tilde{H} \cap U_{i}$. By Corollary 2.4 this means that there is a decomposition factor of $\pi^{*}\left(M_{\alpha}^{\beta}\right)$ with support on $\tilde{H}$.

Corollary 5.3. The module $\pi^{*}\left(M_{\alpha}^{\beta}\right)$ is irreducible if and only if

$$
\beta_{1}, \ldots, \beta_{m}, \sum_{i=1}^{m} r_{i}^{1} \beta_{i}, \ldots, \sum_{i=1}^{m} r_{i}^{s} \beta_{i} \in \mathbb{C} \backslash \mathbb{Z} .
$$

We can use this and the decomposition theorem to get a sufficient criterion for when $M_{\alpha}^{\beta}$ is irreducible. We will use the decomposition theorem in the general form stated by Kashiwara [11] and recently proved by Mochizuki [12. (Our module is regular, so our application actually could have referred to old versions of the decomposition theorem.)

Theorem 5.4. Assume that the $r_{j}^{i}$ are defined by the property (5.1) of a resolution of the singularities of $V(\alpha)$. Then $M_{\alpha}^{\beta}$ is irreducible if $\beta_{1}, \ldots, \beta_{m}, \sum_{i=1}^{m} r_{i}^{1} \beta_{i}$, $\ldots, \sum_{i=1}^{m} r_{i}^{s} \beta_{i} \in \mathbb{C} \backslash \mathbb{Z}$.

Proof. Given the condition in the theorem, by Corollary [5.3, $\pi^{*}\left(M_{\alpha}^{\beta}\right)$ is irreducible. Consider now the following pullback diagram:

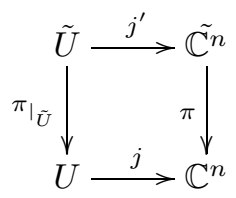

where $U=\mathbb{C}^{n} \backslash V(\alpha)$ and $j^{\prime}$ and $j$ are the respective inclusions. By definition $M_{\alpha}^{\beta}=j_{*}\left(M_{\left.\alpha\right|_{U}}^{\beta}\right)$. Since $j$ is an affine map, this remains true interpreted in the derived category $D\left(D_{\mathbb{C}^{n}}\right)$, where $j_{*}$ now is the derived functor of $\mathcal{O}_{\mathbb{C}^{n}}$-modules (which is also the direct D-module image $j_{+}$). Similarly $j_{*}^{\prime}$ and $\pi_{\left.\right|_{\tilde{U}} ^{*}}^{*}$ are exact 
functors, the latter since $\pi_{\left.\right|_{\tilde{U}}}$ is an isomorphism. By Theorem 1.7.3 of [10], pullbacks behave nicely in the derived category $D\left(D_{\tilde{\mathbb{C}}^{n}}\right)$, so

$$
\pi^{*} M_{\alpha}^{\beta}=\pi^{*} j_{*}\left(M_{\left.\alpha\right|_{U}}^{\beta}\right)=j_{*}^{\prime} \pi_{\left.\right|_{\tilde{U}}}^{*}\left(M_{\left.\alpha\right|_{U}}^{\beta}\right),
$$

and consequently

$$
H^{r} \pi^{*} M_{\alpha}^{\beta}=0 \text { if } r \neq 0 .
$$

Now, in the derived category,

$$
\pi_{+} \pi^{*}\left(M_{\alpha}^{\beta}\right)=\pi_{+} j_{*}^{\prime}\left(\pi_{\left.\right|_{\tilde{U}}}^{*}\left(M_{\left.\alpha\right|_{U}}^{\beta}\right)\right)=j_{*}\left(\pi_{\left.\right|_{\tilde{U}}}\right)_{+} \pi_{\left.\right|_{\tilde{U}}}^{*}\left(M_{\left.\alpha\right|_{U}}^{\beta}\right)=j_{*}\left(M_{\left.\alpha\right|_{U}}^{\beta}\right)=M_{\alpha}^{\beta} .
$$

But by the Decomposition Theorem (see e.g. [12]), $H^{0} \pi_{+} \pi^{*}\left(M_{\alpha}^{\beta}\right)$ is semisimple. However $M_{\alpha}^{\beta}$ is indecomposable, since it is rank 1 over the generic point and torsion free as a $\mathbb{C}[x]$-module, and hence it is irreducible.

Remark 5.5. We do not know whether this criterion is necessary, i.e. if $M_{\alpha}^{\beta}$ irreducible implies that $\pi^{*} M_{\alpha}^{\beta}$ is irreducible too. See Corollary 6.2 for an example of a case where the criterion is both necessary and sufficient.

Example 5.6. Consider $\mathbb{C}^{n}$ and the hyperplane arrangement given by the big diagonal; i.e., let $\alpha=\prod_{1 \leq i<j \leq n}\left(x_{i}-x_{j}\right)$. Set $\alpha_{i j}=x_{i}-x_{j}$ and call the corresponding divisor $D_{i j}$. Consider the module $M_{\alpha}^{\beta}$, where $\beta=\left(\beta_{i j}\right)$. Fulton and MacPherson [8, 7] have constructed a resolution of singularities in this case, which satisfies i) and ii) above. This is a map $f: Y \rightarrow \mathbb{C}^{n}$ with the following property ([8, Theorem 4]). To each subset $S \subset\{1, \ldots, n\}:=[n]$ there is a divisor $E_{S}$ such that schemetheoretically $f^{-1}\left(D_{i j}\right)=\sum_{\{i, j\} \subset S} E_{S}$. The divisor $E_{i, j}$ is the proper transform of $D_{i j}$. In the terminology of the theorem this means that $r_{S}^{i j}=1$ or 0 , according to whether $S$ contains both $i, j$ or not. Hence by the previous theorem, $M_{\alpha}^{\beta}$ is irreducible if for all $S \subset[n]$ we have that $d_{S}=\sum_{\{i, j\} \subset S} \beta_{i j} \in \mathbb{C} \backslash \mathbb{Z}$.

\section{Plane case}

In this section we will exemplify the preceding by describing the pullback to a resolution of singularities of the $A_{2}-$ module $M_{\alpha}^{\beta}=\mathbb{C}[x, y]_{\alpha} \alpha^{\beta}$, where $\alpha=\prod_{i=1}^{m} \alpha_{i}$, $\alpha_{1}=x, \alpha_{2}=y$ and $\alpha_{i}=x+c_{i} y$ for all $i=3, \ldots, m$ and $c_{i} \neq c_{j}$ for all $i \neq j$ and $\beta=\left(\beta_{1}, \beta_{2}, \ldots, \beta_{m}\right) \in \mathbb{C}^{m}$. We only need to blow up the origin. This is the locus:

$$
\widetilde{\mathbb{C}}^{2}=\left\{(x, y),\left[W_{0}, W_{1}\right]: x W_{1}=y W_{0}\right\} \subset \mathbb{C}^{2} \times \mathbb{P}^{1},
$$

together with the map

$$
\pi: \tilde{\mathbb{C}^{2}} \longrightarrow \mathbb{C}^{2},
$$

which is the restriction of the projection of $\mathbb{C}^{2} \times \mathbb{P}^{1}$ onto the first factor. Let $U_{1} \subset \tilde{\mathbb{C}}^{2}$ be the open subset given by $W_{0} \neq 0$. In terms of Euclidean coordinates, $w_{1}=\frac{W_{1}}{W_{0}}$ and we can write

$$
U_{1}=\left\{(x, y),\left(w_{1}\right): x w_{1}=y\right\}=\left\{\left(x, x w_{1}, w_{1}\right)\right\} \subset \mathbb{C}^{2} \times \mathbb{C}^{1} .
$$

From this description we see that $U_{1} \cong \mathbb{C}^{2}$ with coordinates $x, w_{1}$. The restriction $\left.\pi\right|_{U_{1}}$ is given by

$$
\pi\left(x, w_{1}\right)=\left(x, x w_{1}\right) .
$$

Similarly let $U_{2} \subset \tilde{\mathbb{C}^{2}}$ be given by $W_{1} \neq 0$, and put $w_{0}=\frac{W_{0}}{W_{1}}$. Then

$$
\left.U_{2}=\left\{(x, y),\left(w_{0}\right)\right): x=y w_{0}\right\}=\left\{\left(y w_{0}, y, w_{0}\right)\right\} \subset \mathbb{C}^{2} \times \mathbb{C}^{1} .
$$



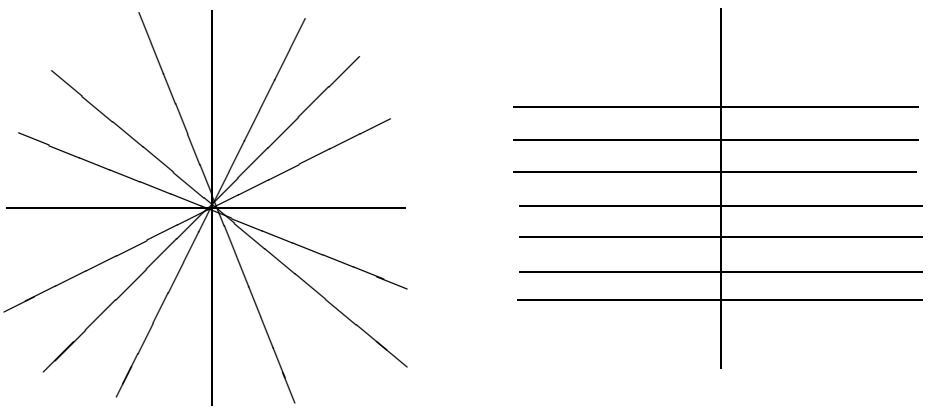

Figure 2. The hyperplane configuration before and after the blowup in one of the affine charts.

So $U_{2} \cong \mathbb{C}^{2}$ with coordinates $y, w_{0}$, and the restriction $\left.\pi\right|_{U_{2}}$ is given by

$$
\pi\left(y, w_{0}\right)=\left(y w_{0}, y\right) .
$$

Clearly $\tilde{\mathbb{C}^{2}}=U_{1} \cup U_{2}$ and hence $\left\{U_{1}, U_{2}\right\}$ is an affine cover of $\tilde{\mathbb{C}}^{2}$. The exceptional divisor $E$ is given in the chart $U_{1}$ by $x=0$, and in $U_{2}$ by $y=0$. (See Figure 2.) Furthermore in $U_{1}$,

$$
\pi^{*}\left(\alpha_{1}\right)=(x), \pi^{*}\left(\alpha_{2}\right)=\left(x w_{1}\right), \pi^{*}\left(\alpha_{i}\right)=\left(x\left(1+c_{i} w_{1}\right)\right), i=3, \ldots, m,
$$

and in $U_{2}$,

$$
\pi^{*}\left(\alpha_{1}\right)=\left(y w_{0}\right), \pi^{*}\left(\alpha_{2}\right)=(y), \pi^{*}\left(\alpha_{i}\right)=\left(y\left(w_{0}+c_{i}\right)\right), i=3, \ldots, m .
$$

Hence, $\operatorname{Div} \pi^{*}\left(H_{i}\right)=\tilde{H}_{i}+E, i=1, \ldots, m\left(\right.$ and $U_{1}=\tilde{\mathbb{C}}^{n} \backslash H_{1}$ and $\left.U_{2}=\tilde{\mathbb{C}}^{n} \backslash H_{2}\right)$. This gives us the numerical description of the resolution that we need to apply Corollary 5.2 .

Proposition 6.1. Consider $\pi_{2}^{*}\left(M_{\alpha}^{\beta}\right)$. Let $I=\left\{i \mid \beta_{i} \in \mathbb{Z}\right\}$ contain $k$ elements, and let $|\beta|=\sum_{i=1}^{m} \beta_{i}$.

(i) If $|\beta| \in \mathbb{Z}$, then there is exactly one decomposition factor with support on each of $\mathbb{C}^{2}, E, H_{i}, i \in I$ and one on each intersection $H_{i} \cap E, i \in I$, and these are all decomposition factors - all together $2(k+1)$.

(ii) If $|\beta| \in \mathbb{C} \backslash \mathbb{Z}$, then there is exactly one decomposition factor with support on $\mathbb{C}^{2}$, and one on each $H_{i}, i \in I$ only-all together $k+1$.

We can compare the criterion for irreducibility of $M_{\alpha}^{\beta}$ that was obtained in Theorem 5.4 with what was proved in the plane case in [1] and see that in the plane it also gives a necessary criterion. As mentioned above, we ignore whether this is true in general. The methods in 1 that prove the following corollary are much more algebraic than those used in the present paper, but might possibly be used to prove necessity, if this is true.

Corollary 6.2. In the plane case, $\pi^{*}\left(M_{\alpha}^{\beta}\right)$ is irreducible if and only if $M_{\alpha}^{\beta}$ is irreducible if and only if $\beta_{1}, \ldots, \beta_{m}, \sum_{i=1}^{m} \beta_{i} \in \mathbb{C} \backslash \mathbb{Z}$.

Proof. See [1]. 


\section{ACKNOWLEDGEMENTS}

We gratefully acknowledge the encouragement of Demissu Gemeda, Addis Ababa University, and dedicate this paper to his memory. We also want to thank Jan-Erik Björk and Rolf Källström for interesting, useful and fun discussions.

\section{REFERENCES}

1. Abebaw, T. and Bøgvad, R., Decomposition of D-modules over a hyperplane arrangement in the plane, Arkiv f. Mat., 48, No. 2, 2010, 211-229. MR2672606

2. Àlvarez Montaner, J., Castro-Jiménez, F. J. and Ucha, J. M., Localization at hyperplane arrangements: combinatorics and D-modules, J. Algebra 316, No. 2, 2007, 662-679. MR2358608 (2008i:16028)

3. Björk, J.-E., Rings of differential operators, North-Holland Publishing Co., Amsterdam, 1979. MR549189 (82g:32013)

4. Björk, J.-E., Analytic D-modules and applications, Kluwer Academic Publishers Group, Dordrecht, 1993. MR1232191 (95f:32014)

5. Coutinho, S. C., A primer of algebraic D-modules, Cambridge University Press, Cambridge, 1995. MR 1356713 (96j:32011)

6. De Concini, C. and Procesi, C., Hyperplane arrangements and box splines, Mich. Math. J. 57 (2008), 201-225. MR2492449 (2010f:52037)

7. De Concini, C. and Procesi, C., Topics in hyperplane arrangements, polytopes and box-splines, Springer-Verlag, New York, 2011. MR2722776 (2011m:52036)

8. Fulton, W. and MacPherson, R., A compactification of configuration spaces, Ann. of Math. (2), 139, No. 1, 1994, 183-225. MR1259368 (95j:14002)

9. Harris, J., Algebraic geometry, Springer-Verlag, New York, 1995. MR.1416564 (97e:14001)

10. Hotta, R., Takeuchi, K. and Tanisaki, T., D-modules, perverse sheaves, and representation theory, Progress in Mathematics, 236, translated from the 1995 Japanese edition by Takeuchi, Birkhäuser Boston Inc., Boston, MA, 2008. MR2357361(2008k:32022)

11. Kashiwara, M., Semisimple holonomic D-modules, Topological field theory, primitive forms and related topics (Kyoto, 1996), Progr. Math., 160, pp. 267-271, Birkhäuser Boston, Boston, MA, 1998. MR.1653028 (99m:32013)

12. Mochizuki, T., Asymptotic behaviour of tame harmonic bundles and an application to pure twistor D-modules, Mem. Amer. Math. Soc. 185 (2007), no. 869; Mem. Amer. Math. Soc. 185 (2007), no. 870. MR2281877 (2007j:32028a) MR2283665(2007j:32028b)

13. Orlik, P. and Terao, H., Arrangements of hyperplanes, Grundlehren der Mathematischen Wissenschaften, 300, Springer-Verlag, Berlin, 1992. MR1217488 (94e:52014)

14. Saito, M., Sturmfels, B. and Takayama, N., Gröbner deformations of hypergeometric differential equations, Springer-Verlag, Berlin, 2000. MR1734566 (2001i:13036)

15. Khoroshkin, S. and Varchenko, A., Quiver D-modules and homology of local systems over an arrangement of hyperplanes, IMRP Int. Math. Res. Pap., 2006. MR2282180 (2009d:32030)

16. Stanley, R. P., An introduction to hyperplane arrangements, in Geometric combinatorics, IAS/Park City Math. Ser., 13, pp. 389-496, Amer. Math. Soc., Providence, RI, 2007. MR2383131

17. Torrelli, T., On meromorphic functions defined by a differential system of order 1, Bull. Soc. Math. France, 132, No. 4, 2004, 591-612. MR2131905(2005m:32015)

18. Walther, Uli, Bernstein-Sato polynomial versus cohomology of the Milnor fiber for generic hyperplane arrangements, Compos. Math., 141, No. 1, 2005, 121-145. MR2099772(2005k:32030)

Department of Mathematics, Addis Ababa University, Ethiopia - And - Stockholm University, SE-10691 Stockholm, Sweden

E-mail address: tabebaw@math.aau.edu.et

Department of Mathematics, Stockholm University, SE-10691 Stockholm, Sweden

E-mail address: rikard@math.su.se 